Je sais bien que les administrations fiscales en France, iguorent enlre elles, mais il serait cependant assez extraordinaire quil me fut impossible d'obtenir de l'enregistrement la bonificalion de tarif parce que la direction des Chemins de fer de l'Etal n'est pas une émanation directe de l'Etat et que je fusse obligé par les contributions directes de payer la patente parce que ces chemins de fer constituent unt administration publique.

II y aurait intérêt, en tout cas, à faire trancher la question pour que l'on sut à quoi s'en tenir.

Paul Bougault,

Avocat à la Cour d'appel de Lyon.

\section{GONDUITES FORCÉES}

\section{IV. - CONSTITtron des conduIIEs.}

les conduits sou: pression sont un organe délicat qui a humé lieu assez fréquemment ì des accidents dont le moindre résultat est d'immobiliser l'usine pour un assez long lemps el qui peuvent parfois amener la destruction d'une partie de l'installation. Il y a donc lieu de surveriller avec le plus grand soin leur établissement.

On les fait en général en acier et parfois, pour les faibles hulls, jusqu'à 40 mètres environ, en béton armé.

On trouvera, dans le deuxième volume du Ccngrès de la Ilouille Blanche de I9Í́, des arlicles très complets, l'un de IV. Auguste Bouchayer, sur les conduites métalliques, l'autre de M. Delanarche'sur les conduites en béton armé. Renvolant donc, pour les détail = complèts, le lectenr à ces deux articles, je me bornerai ici à des renscignements généraus.

Conduites en béton armé. - Ce sont des ouvrages de héton armé, convenant particulièremnt aux faibles chute: et aux gros débits. On arrive couramment à $3 \mathrm{~m}$. 50 et 4 mètre, de diamètre. Elles sont armées, parfois, avec des aciers en spirale, le plus souvent avec des directrices circulaires convenablement espacées et reliées par des génératrires rectilignes. Le point important réside surtout dans lo dosage ef la mise en œuvre du béton de manière à nbtenir des ouvrages étanches ne laissant pas filtrer l'eau ( $\left.{ }^{1}\right)$. On peut d'ailleurs, à ce point de vue, employer des procédés dérivés du système Bonna, qui consiste à doubler intérieurement la conduite d'une tôle mince de I à $2 \mathrm{~mm}$. d'épaisseur, insuffisante pour résister à la pression (et qui pourrait d'ailleurs s'écraser sous son propre poids), mais qui donne l'étanchéité. On sépare ainsi nettement la partie destinée à empêcher les filtrations d'eau de celle qui donne la résistance

Conduites en acier. - La technique de ces ouvrages est aujourd'hui bien fixée et va faire l'objet d'un cahier des charges type dont la discussion est très avancée et qui sera publié par la Société hydrotechnique de France.

J'en indiquerai les particularités principales. Ces conduitesont soudées ou rivées. T.e métal à employer doit être analogue à celui employé par les Compagnies de Chemins de fer pour les chaudières. On distingue deux qualités principales.

$I^{\circ}$ Tôle chaudière qualité A qui sera probablement dési-

\footnotetext{
(1) Cies filtrations, en dehors des pertes d'eau, auraient l'ınconvénıent d'amener l'appauvrissement progressif des bétons.
}

gnée sous le nom de tôle dacier soudable pour conduites forcées qualité $\mathbf{A}$. Ses caractéristiques mécanique-sont les suivantes.

$$
\begin{aligned}
& \text { R : } 35 \text { à } 40 \text { Kgsimm². } \\
& \text { Al : } 30 \%
\end{aligned}
$$

Cette qualité doit ètre nécessairement emplosée pour toutes les conduites soudée, et pour toutes les pièces spéciales (coudes par exemple) rivées ou soudées subissant un travail de forge. En dehors des e-sais ordinaires, on doit faire subir à ces tòles un essai de soudabilité.

$2^{\circ}$ Tôle chandière qualité B - ou hole diacior pour conduites forcées qualité B. -- Qucique la qualité A soit fonjours meilleure, on pourra se contenter de celle tòle pour les viroles droites rivées. Ses canactéristiques mécaniques sont :

$$
\begin{aligned}
& \mathrm{R} \cdot 40 \text { à } 16 \mathrm{kgs} / \mathrm{mm}^{2} \\
& \mathrm{Al}: 27 \%
\end{aligned}
$$

Le recuit des tòles est indispensable.

La soudure se fait à l'autogène par martelage of parfois avee addition de barres de fer de suède pour les forte- épaiseurs. Pour la rivure, il convient, an-dessus de ro $\mathrm{mm}$. d'épaisseur, de ferer entierement le tron. Au-dessous de I mm., on peut admellere le poinçonnage, ì condilion d'aléser le trou en augmentant le diamètre de poinçomnage $\mathrm{d}$ au moins $5 \mathrm{~mm}$.

Le point essentiel est d'éviter l'emploi des aciers fragiles.

Les joints transversaux des conduites peunul of faire suivant plusieurs systèmes dont le détail est demné dan. l'article précité de M. Bouchayer. Pour le- conduites rivées, on s" sert en général de grandes ef de petites viroles emboîtéc: successivement l'une dans l'autre et rivées suirant do directrices. La seule difficulté consiste dans la pince ì ménager à la jonction des clouures longitudinales ef transversales. En tous cas, il faudra toujours un matage soigné des joints.

Pcur le: conduites soudées, étant donné que l'efiort sui. vant les directrices est la moitié de celui suivant los génć. ratrices, on pourra, dans certains cas, sans rencontrer ici la difficulté due à la pince, assembler le, viroles par rivure: mais le plus souvent, on utilisera des joints à brides aver bagues annulaires en caoutchonc: il faudra. dans ox cas, que ces bagues no puissent pas être chassées par la pression, ni écrasées au moment de la confection du joint.

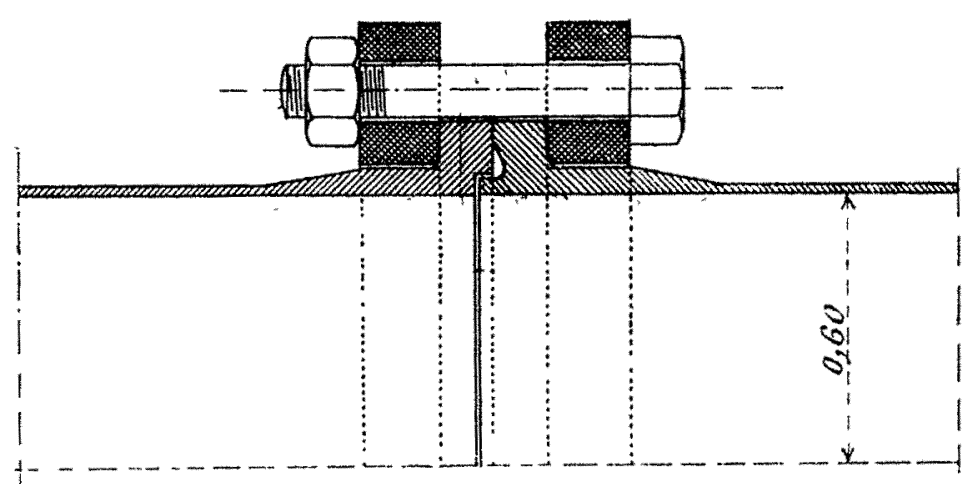

Fig. 4

On trouvera sur la figure f́ un dessin des joints des conduites de Soulom qui remplissent ces conditions of ont donné, en service, toute satisfaction. lev brides sont assemblée - ur les viroles par des soudures.

I'épaisseur des tôles doit ètre calculée de façon que leffort 
moléculaire, sous la pression $y+a b$ ne dépasse pas $8 \mathrm{kgs}$ par $\mathrm{mm}^{2}$. Les soudures seront caloulées pour les $9 /$ io de ce chiffre. En cas de conduites rivées, il faudra tenir compte de la diminution de section due à la rivure.

A cause de la nécessité de mettre un nombre suffisant de rivets, on arrive assez vite à l'obligation d'une triple clouure et la rivure ne peut plus être appliquée au delà de 22 à $25 \mathrm{~mm}$. d'épaisseur de tôle.

II me reste à indiquer les limites industrielles. On ne descend pas, en pratique, au-dessous d'une épaisseur de 4 à $5 \mathrm{~mm}$.

Quant aux conduites soudées, on pent scuder des épaisseurs de 3\% nm. et à l'usine des Sept-Laux, qui représente une différence de niveau de 1.000 mètres environ entre les extrémités amont et aval des conduites, on est même arrivé ì constituer des fuyaux ayant, à la base, o m. 52 de diamètre intérieur et $3.1 \mathrm{~mm}$. d'épaisseur.

On a pu, avec la sondure, réaliser des pièces très importantes et parmi lesquelles on peut citer, je crois, celles fournies par la maison Bonchaver à la Compagnic du Midi pour

\section{V. - Dispositions piVERSES DEs CONDUITES ForcéEs.}

Conduites à diamètre variable. - Les conduites étant calculées par la formule connue $: R=\frac{p D}{2 e}$, où $\mathrm{R}$ est l'effort moléculaire admis pour le matériau qui constitue la conduite, $D$ son diamètre intérieur mesuré sur la plus grande virole (point à retenir quand il s'agit de conduites rivées), $p$ la pression donnée par l'expression $y+a b$ et $e$ l'épaisseur, on voit que lorsqu'on arrive au voisinage du maximum d'épais. sour admissible, D prend une valeur conmmandée. Dans le cas des hautes chutes, on ne peut obtenir ainsi qu'une valeur de D assez petite qui, maintenue sur toute la longueur de la conduite, donnerait lieu à des pertes de charge considérables. On arrive alors à concevoir des conduites coniques, ou, pour mieux dire, télescopiques à diamètre décroissant de la base au sommet. A mesure qu'on arrive, pour un diamètre donné, à une épaisseur peu pratique, on passe en descendant à un diamètre plus petit. Si cette variation esl faible, on la lattrapera sur un joint. Sinon la jonction se

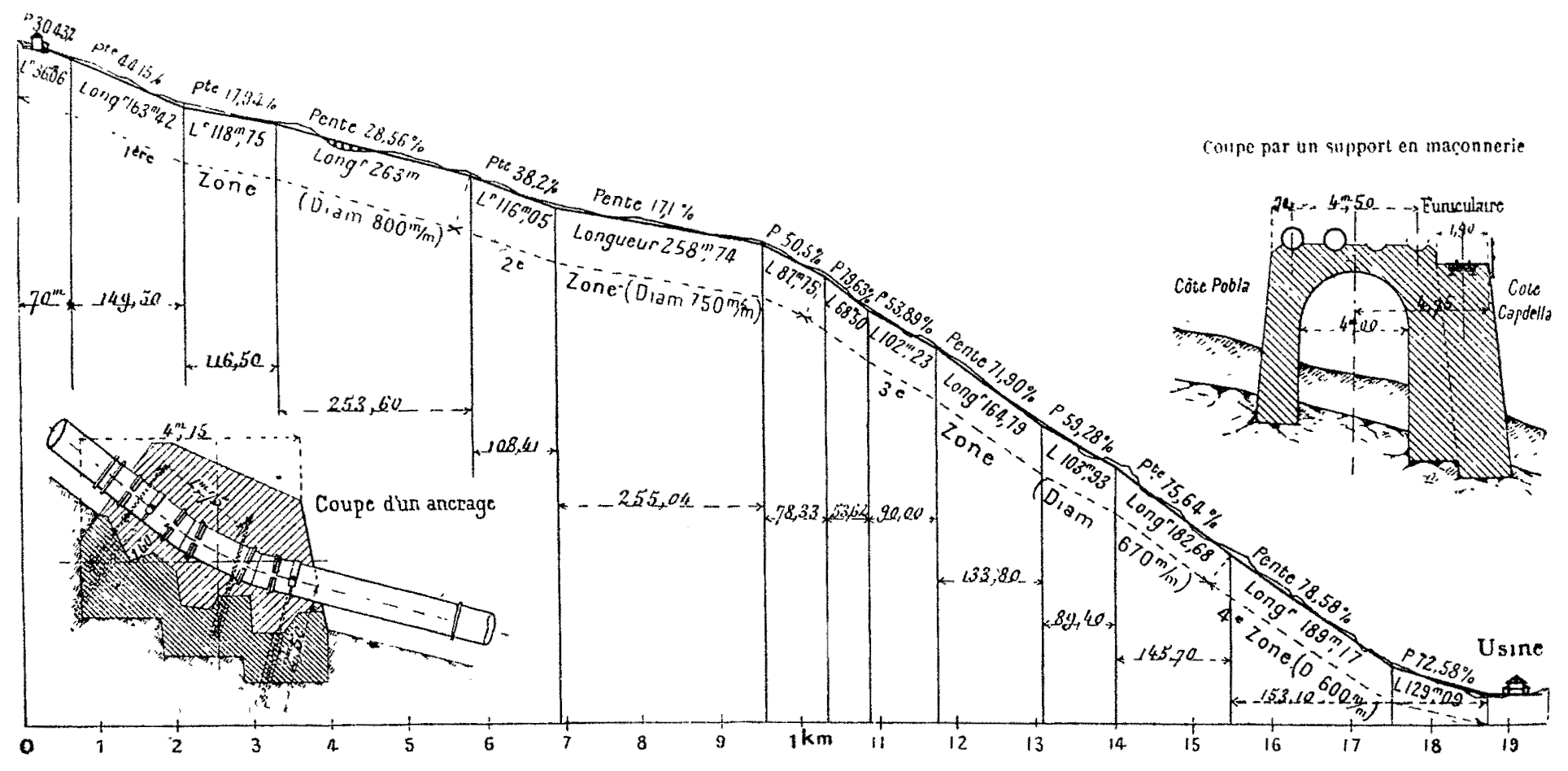

Frg. 5

l'usine de Sculom. Les tromgons alteignent $18 \mathrm{~m}$. 50 de longueur entre brides et l'épaisseur arrive à $15 \mathrm{~mm}$. pour les ronduites de $\mathrm{j}$ 11. 200 de diamètre intérieur et à $20 \mathrm{~mm}$. pour des conduites de o $\mathrm{m}$. 8. En dehors des soudures longitudinales, chaque tronȩon comprend deux soudures transversales pour assemblage de viroles de $6 \mathrm{~m}$. de longueur et les soudures des brides d'about. C'est le maximum de longueur atteint jusqu'à ce jour à cause de la longueur des bras des machines à souder.

Il faut reconnaître que ces longueurs ne sont pas toujours pratiques à cause des difficultés de transport et de pose et, pour l'usine d'Eget. on a dî réduire la longueur des troncons à ra mètres.

Un dernier procédé, d'origine allemande, a été emplové à l'usine de Fully (Valais) où la chute brute réalisée atteint lc rhiffre énorme de 1.650 mètres. II dérive du système Mannessmann et consiste à poinçonner à chaud un lingot d'icier qu'on transforme ainsi pen à peu en conduite. Co procédé est resté exceptionnel ef n'est pas entré dans la pratique courantc. fera à l'aide de pièces de chaudronneric spéciales de forme conique.

Je donne ci-dessous, à titre de renseignement, les caracléristiques et le schéma (fig. 5) des conduites de l'usine de Capdella, dońt j'ai déjà parlé.

Chute brute totale : 830 mètres.

Débit maximum par conduite : 1.750 litres.

Vitesse admise : de $3 \cdot \mathrm{m}$. 50 dans les troncons de diamètre à $5 \mathrm{~m}$. 5o pour ceux de $\mathrm{o} \mathrm{m}$. 600 de diamètre.

Perte de charge pour le débit de 1.750 litres : $5,3 \%$, soit: 45 mètres

Si l'on fait le calcul de cette conduite, en prenant $\alpha b=0,20 y$; soit $p=1,20 y$, on verra, au point de vue de l'effort moléculaire, qu'elle est de construction plutôt hardíe.

Je donnerai également les caractéristiques et le schéma (fig. 6) des conduites de l'usine d'Artouste projetée dans la Haute Vallée d'Ossau pour la Compagnie du Midi.

Chute brute totale : $790 \mathrm{~m}$.

Débit maximum par conduite : r.o8n 1./

Débit moyen par conduite : $5.401 . / \mathrm{s}$. 
Nombre de conduites : 3 .

litesse maximum admise : de 2,15 , dans les troncons de o $\mathrm{m}$. 800 de diamètre à $3,8 \mathrm{r}$ pour ceux de 0,600 de diamètre.

$a b$ à la base en régime de marche maximum : $83 \mathrm{~m}$.

Perte de charge totale : en débit moyen $7 \mathrm{~m}$. 54 , soit I $\%$. Perte de charge totale : en débit maximum $32 \mathrm{~m}$. 60 . so 1 h, I \%.

Chute nette à la base de la conduite forcéc:

En régime moyen : 782,46 .

En régime maximum : 757,40 .

Si l'on avait conservé sur toute la longueur le diamètre de 0,600 , réalisé à la base, les pertes de charge auraient atteint respectivement les chiffres de $13 \mathrm{~m}$. et $58 \mathrm{~m}$. 6o, soit $\mathrm{x}, 6 \%$ et $7,4 \%$.

$\mathrm{Au}$ point de vue du calcul de $b$, on peut, pour les conduites à diamètre variable, prendre une expression dérivant
C'est ainsi que la maison Joya, de Grenoble, a construit les conduites de la chute de Villeneuve d'Aoste. L'ne première conduite de $1 \mathrm{~m}$. oło de diamètre se bifurque en deux antres de o m. 740. Ghacune de celles-ci se bifurqua encore $\mathrm{nn}$ deux autres, de o m. 540.

Je donne ci-après (fig. 7) le dessin de la bifurcation de la conduite de $\mathrm{r} \mathrm{m}$. olıo en deux de o $\mathrm{m}$. 74o. On y remarquera les robinets-vanne-permettant d'isoler du tronc commun chacune des deux conduites inféricures, pour les cas d'arrì on de réparation.

Les pièces de jonction dites "culottes" sont des piècespéciales de chaudronnerie-ront la fabrication doit être particulièrement soignée.

On remarquera que les départs des bifurcations, -ur la. conduite de Villeneuve d'Aoste, șe font à $55^{\circ}$. Bien que cette installation ait donné d'excellents résultats, il faudra tou-

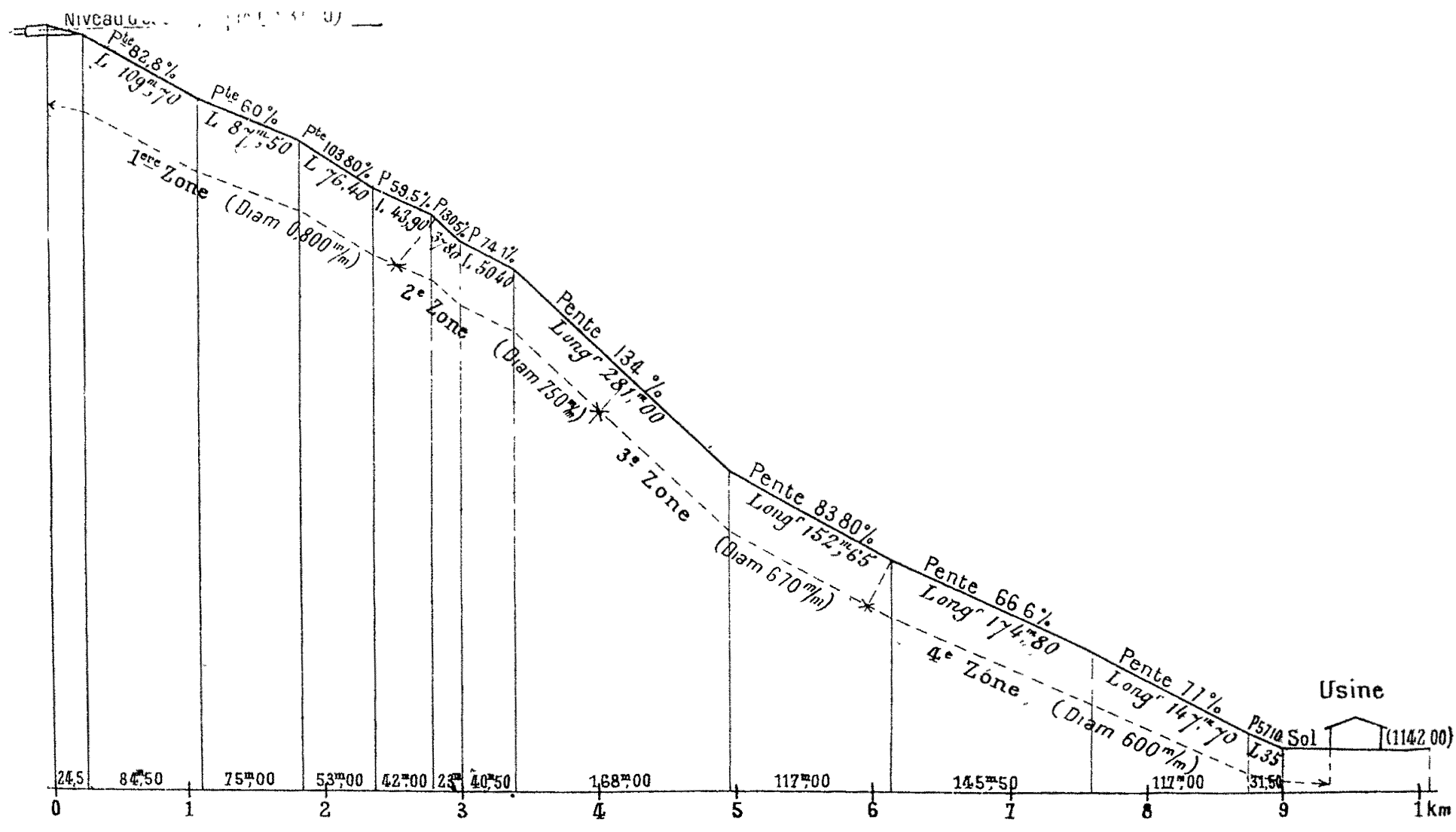

Fig. 6

d'une ancienne formule, dite formule de Michaud, qui s'érril avec les notations précédemment adoptées.

$$
b=\frac{2 L v}{g T}
$$

Dans le cas qui nous occupe, elle s'écrira :

$$
b=\frac{2 \Sigma \mathrm{L} v}{g \mathrm{~T}}
$$

L et $V$ étant mesurées par tronçons successifs.

Les résultats obtenus en pratique seront très suffisamment exacts.

La détermination rationnelle des longueurs et des diamètres des divers tronçons au point de vue économique a donué lieu à diverses études. En général, cette détermination se fail plutôt par tâtonnements et de façon empirique.

Conduites bifurquées. - On est arrivé dans le même ordre d'idées et pour obtenir des solutions économiques, à réaliser des conduites bifurquées dans lesquelles on fait varier à mesure qu'on descend, non seulement le diamètre, mais encore le nombre des conduites. jours prendre garde aux déformations qui peuvent se produire, lors de la mise en charge, dans ces assemblages obliques et à la difficulté de calculer les efforts moléculaires au voisinage des courbes d'intersection. Il semblerait préférable de réaliser ces assemblage: en faisant arriver la conduite dérivée normalement à la conduite principale, quitte à rétablir le parallélisme des conduites en redressant la conduite dérivée par une courbe de petit rayon dont la présence no constitue pas un inconvénient sérieux. Le calcul du coup de bélier dans ces conduites se fera comme pour les conduites à diamètre variable.

Je donne également, à titre de renseignement, le schéma (fig.- 8) des conduites étudiées pour une usine projetée à Saillens (Haute Vallée de l'Arriège) pour la Compagnic des Chemins de fer du Midi.

Les caractéristiques sont les suivantes

Chute brute totale : $7 \times x$ mètres.

Débit maximum total : 3.600 mètres.

Vitesse maximum admise: dan: le trone commun : 2 m. $9^{3}$. 
Vitesse mavimum admise - à la base des petites conduites 4 m. 85 .

On remarquera que l'on a à l'orıgine une conduite unique dont le diamètre est 1 III. \). Quand l'épaisseur deviendrail trop forte, elle - divise en deux parties distinctes telles que le débit de l'une est la moitié de celui de l'atutre. Enfin wite dernière se bifurque en deu, équivalant chacune à colle qui s est déjà détaillér.

Le diamètre de chaque conduite à la base est de o m. 560 . Près de l'usine, ces trois conduites se raccordent les uncs aux autres de façon ì pouvoir alimenter indistinctement.les groupes de 5.000 poncelets sur les aubes des turbines qui constituent l'armoment de liusine.

la perte do charge prévuc en négime maximum est égale $\therefore$ \{o,1, , wit $5,-7 \%$ de la chute brute.
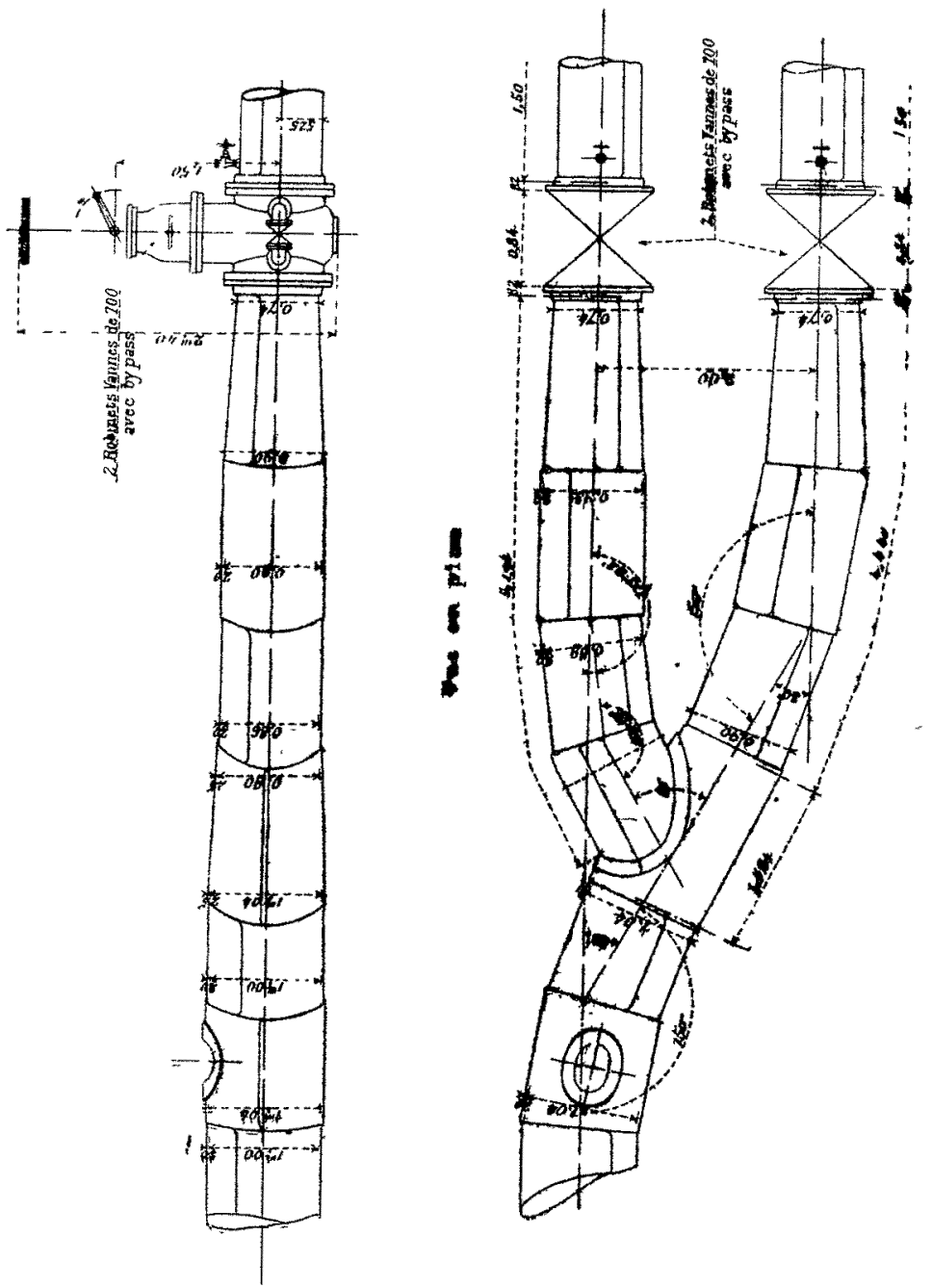

Fir. 7

\section{Vl. - Cirol rement des condijtes. - Collegteurs.}

line question importante est celle du nombre et du groupement des conduites. Elle est intimement liée à la répartition du nombre et de la puissance des groupes générateurs ì établir à l'usinc. Cette question a donné lieu récemment à deux notes parues au $C, R$, de l'Académie des Sciences et présentées par M. Baticle, ingénieur des Ponts et Chaussées (1), qui a cherché, pour les installations hydrauliques, une formule analogne à celle de Thomson pour l'électricité.

La solution dépend nón seulement du point de vue économique, mais encore du service que l'on veut assurer et de la sécurité que l'on veut obtenir.

Ainx usines de Soulom et d'Eget de la Compagnie du Midi,

(1) Voir n* Mars Avrll 1919 de la " Houille Blanche" qui étaient les premières et qui ont été établies à un momen! où l'on ne prévoyait pas l'extension acluellement admise de la production du couranl, on a établı des groupes indépelldants, composés chacun d'une conduite, d'une turbine cl d'un alternateur, ainsı que des accessoires correspondants. Dans chaque usine, existe un groupe semblable de réserve, de lelle laçon que chaque groupe peut ètre arrêté à tour du ròle et soumis à un entretien méthodique pour éviler les pannes et les avaries ré,ultant d'un service trop prolongé.

A l'usine de Campocologno, dans les Grisons, chaque conduite alimente un groupe délini de turbines.

Dans la plupart des usines, les conduites sont reliées ì ut: conduite unique, dite collecteur, sur laquelle elles sont toutes montées en parallèle et qui alimente indifléremment toutes les turbines. Le nombre des conduites est ainsi sans aucune relation avec celui des turbines.

Cette disposilion est arantageuse en ce qu'on peut choisir pour ces deux séries d'organes, indépendamment l'un de l'autre, le groupement le plus économique. Mais elle fait dépendre l'ensemble d'une pièce délicate, le collecteur, dans lequel se déversent toutes les conduites; de plus, elle exigc l'interposition entre collecteur et conduite dun robinet de fabrication difficile et coùteuse, mais nécessaire si l'on ne veul pas qu'une avarie à une conduite immobilise toute l'usine.

Organes spéciaux aux conduites. - En dehors de la conduite proprement dite, il est indispensable de prévoir un certain nombre d'appareils. L'emploi d'un reniflard ou de ventouses au départ de la chambre de mise en charge pour éviter l'aplatissement dû au vide produit par la vidange trop rapide d'une conduite en cas de rupture en bas est connu. Mais un fait moins connu est celui des surpressions locales très dangereuses dues à des poches d'air isolées. M. de Sparre a montré par le calcul que ces surpréssions peuvent dépasser la pression statique. M. Camichel a d'ailleurs pu se rendre compte, dans des essais récents, que la formation de ces poches d'air, puis leur départ brusque, au cours d'un remplissage de conduites, peut donner lieu en certains points ì des surpressions parfois supérieures à la pression statique.

Il sera donc bon de ménager de petits robinets de purge à chaque point où peltvent se produire des accumulations d'air, aux points hauts en particulier, ou sur le parcours des zones où la conduite est presque horizontale.

Des robinets de vidange sont également à prévoir à tous les points bas pour évacuer les boues.

Enfin, il ne faut pas oublier que les gros robinets d'arrêt des conduites, qui sont de grande dimension et fonctionnent sous forte pression, sont très difficiles à manóuvrer. Ils doivent toujours être munis de by-pass permettant l'égalis lliin des pressions sur les deux faces et, en dehors de la manœuvre à main, toujours nécessaire, mais très lente, il est d'une bonne pratique de prévoir une manouvro mécanique, soil par servo-moteur mécanique, soit par servo-moteur hydail lique, soit par moteur électrique.

Cette remarque -s'applique aux robinets-vannes des turbines.

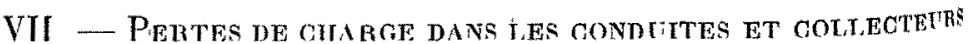

Une question importante o-t collo des pertes de charge dans les conduites forcérs.

On les calcule en général en extrapolant, pour les grosses conduites modernes, les formules de Flamant qui ont donné des résultats suffisants.

La question serait donc suffisamment connue s'il n'y avail 
licu de tenir compte, dans certains cas, des pertes singulières signalées par M. Bouchayer à la Société hydro-technique de France.

Ln dehors, en effet, du terme $\frac{v^{2}}{2} g$ dù à la mise en vitesse de l'eau dans le réservoir amont qui peut avoir son importanre dans les basses chutes et qu'on ne récupère qu'en partic à l'entrée des turbines, de la perte qui se produit quand l'eau arrive dans le canal de fuite (théorème de Bélanger), il peut y avoir, surtout dans les collecteurs, des pertes singulières, dont je ne dirai que quelques mots, la question n'étant pas cncore ćlucidée. Flle est liée à une autre question, d'actualité immédiate, consistant à ajouter de nouvelles turbines sur des conduites et un collecteur donnés pour mieux utiliser les hautes eaux.

Voici comment s exprime à ce sujet M. Bouchayer :

"Etant donnée ume conduite alimentant une turbine, si

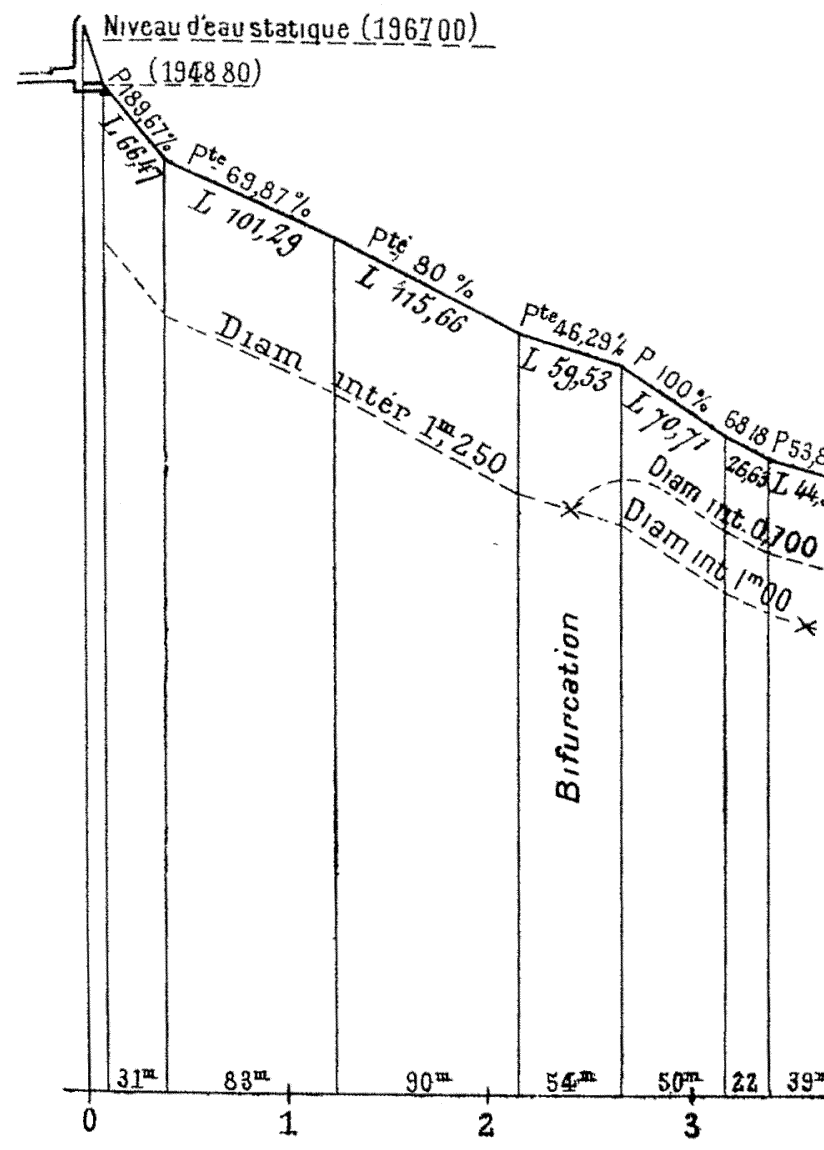

ou installe sur cette conduite une deuxième turbine identique à la première, c'est-à-dirc si on double l'ouverture du distributeur, le débit de la conduite est très augmenté.

"Il serait doublé si la perte de charge était nulle. Comme cela n'est pas, il est facile de voir que si on désigne par :

« H la chute brute;

"Q le débit primitif et $h$ la perte de charge correspondante ;

" $Q^{\prime}$ le débil pour l'ouverture du distributeur doublée. le nouveau débit s'cxprime par la formule :

$$
\mathrm{Q}^{\prime}=2 \mathrm{Q} \sqrt{\frac{\mathrm{H}}{\mathrm{H}+3 h}}
$$

la perte de charge $h$ étant supposée varier comme le carré do la vitesse de l'eau dans la conduite.

" $Q^{\prime}$ n'est le double de Q que lorsque $h$ est nul ; si $h$ est les $5 \%$ de $\mathrm{H}$, on a $\mathrm{Q}^{\prime}=\mathrm{r}, 86 \mathrm{Q}$, ce qui correspond à une perte de charge 3,45 fois plus grande que la première, c'est-à-dirr à $r, 2 \%$ de la chute brute au licu de $5 \%$.

"La perte de charge est done fort sensible.

"J'ai constaté à la Société de l'Fau d'Olle (Usine du Vernay) avant qu'on ail modific le collecteur, une diminution importante de la puissance de turbines à mesure qu'on allait vers l'aval du collecteur.

"Pour 5 turbines du mème lype, la puissance unitaire passait de $P$ à $\frac{P}{2}$ en décroissant régulièrement de la premièro (ì l'amont) à la dernière (la plus aval).

"Cette constatation de différences de rendement de turbines identiques montre d'une façon péremptorre que toute la question est liéc à celle des pertes singulières. I.es remous désastreux constatés à l'u-ine du Vernay provenaient simplement de ce que les branchements aux turbines étaient d'un diamètre trop petil: lorsque la vitesse augmentail il se produisait un tel remous de péuétration dans les branchements que l'alimentation des turbines aval se faisail difficllement et avec des pertes singulières considérables. On a alors modifié complètement le collecteur, angmenté le diamètre des lubulures de prise pour réduire la rilesse d'entrée dans ces tuyaux et retrouver ainsi les conditions d'un fonctionnement normal. 
“ Yous nous borneron. à rappeler le résultat d'après lequel cette puissance est obtenue lorsque la perte de charge attent le ticrs de la chute brute, la seule hypothèse qu'il comporte étant celle de la proportionnalité des pertes de charge au carré de la vitesse de l'eau dans la conduite.

" Eu égard à ce "résultat, si on veut tenir compte de ce quatux grandes vitroses les perles singulières sont susceptibles de croitre d'une façon anormale, nous pourrons conclure qu'il ne faut pas craindre, en observant les précautions de réglage indiquées par M. le Contte de Sparre, d'utiliser le débit possible jusqu'a la perte du quart de la chute brute. "

Les précaulions de réglage indiquées par $M$. de Sparre consi,tent à avoir une fermeture du distributeur dallure non linéaire, mais ralentissant de plus en plus à nesure quion s'approche de la fin de la manœuvre.

Remarquons d'aillours que le fait d'arriver à une pert. de charge égale à $25 \%$ de la chute est exceptionnel ct qu'en pratique, on se borne encore aux chiffres que j’åi précédemment indiqués.

J'en ai ainsi terminé avec la question des conduites, sauf́ un point de détail. Il convient de protéger les conduites contre le gel ; dans certains cas, on y arrive en les recouvrant de terre, mais la surveillance et l'entretien sont moins faciles. Si l'on préfère les laisser à découvert, il faudra avoir soin, quand elles sont pleines, d'éviter les arrêts d'eau en temps froid ef d'y maintenir toujours un léger écoulement. En par. ticulier, il faudra toujours laisser un peu ouverts les robinets de vidange ou autres, aux abords desquels il y aurait, sans cette précaution, une zone d'eau morte où la glace pourrait se former

\section{Eydoux,}

Professeur suppléant à l'Ecole nahonale des Ponts et Chaussées (1).

\section{L'AMÉNAGEMENT DU RHONE}

Dans notre dernier numéro nous avons annoncé la publication d'une note de $M$. de la Brosse, relative à l'aménagement du lac Léman. Nous donnons ci-après ce travail d'un intérêt évident :

\section{Note sur l'Utilité d'une Augmentation d'Amplitude dans les Variations de Niveau du Lac Léman}

Dans mes précédents rapports sur l'aménagement du Rhòne (Comparaison des puissances réalisables par les projel de Bellegarde-Malpertuis et de Génissiat, Importance et Valcur économique de l'énergie du Rhône), j'ai eu plusieurs fois l'occasion de signaler, sans m'y arrêter, l'intérêt que pourrait avoir un relèvement do niveau du lac Léman ou, plutòt, un accroissement de sa capacité comme réservoir rég'ulateur du débit du Rhône par une augmentation d'amplitude de ses variations réglementaires.

Celles-ci sont restées longtemps assez arbitraires, elles n'ont été bien définies que par une convention intervenue lo 17 décembro r 88/ après de longues et laborieuses négociations entre les états de Genève, Yand et Valais, sans partiripation de la France. Ans termes de l'article la de cette

(1) Dans notre dernior numéro. une onission nous avait fail écrire 1) Eydous, professeur à l'ficole nationale des Ponts of Chaussíses M. Eydonx nous prie de faire la rectification suivante: Professeu suppléant l'Ecole nationale des Ponts et Chaussées convention, les barrages de Genève doivent ètre mancuvrés de l'açon à maintenir le niveau du lac entre les cotes 1,30 el 1,go en contre-bas du repère du Niton $\left({ }^{1}\right)$, lamplitude des variations de niveau est ainsi limitée à $\mathrm{om}$. 6o, le niveau moyen du lac placé à $x \mathrm{~m}$. 6o en contre-bas du repère $P$. N., c est-ì-dire à l'altitude $37^{2} \mathrm{~m} .02$, les écarts de part el d'autrc de celte moyenne ne devant pas dépasser o m. 3o.

La superficie du lac Léman est . un peu supérieure à 580 kilomètres carrés environ $582 \mathrm{~km}^{2}$,4) en sorte que la tranche de o 11 . 6o qui peut jouer comme réserve pour accroitre ou, modérer le débit du fleuve représente $582.400 .000 \mathrm{~m}^{2} \times 0 \mathrm{~m}$. $60=349.440 .000$ mètres cubes, soil en chiffre rond 350 millions de mètres cubes, grâce à quoi et avec des manceuvres convenables en temps opportun on arrive assez bien à maintenir dans le Rhône pendant la sáson des basses eaux (de décembre à mars), un débit de I $20 \mathrm{mc} . / \mathrm{s}(2)$.

Pendant la guerre, les besoins de force hydraulique s'ćtant accrus partout même chez les neutres, à cause de la pénurie générale du charbon, le Conseil Fédéral consentit à laisser relever exceptionnellement dé o $\mathrm{m}$. r5 le niveau maximum du lac dans l'hiver rgr-igr8 et abaisser de $0 \mathrm{~m}$. 20 son niveau minimum du 55 mars au 15 avril 1918 . C'était un innovation importante que les riverains des cantons de Vaud ef du Valais ont pu voir avec quelque appréhension, mais qui, en somme, ne paraîl pas avoir mal réussi et qui a démontré par le fait qu'il n'était pas impossible de revenir sur la Convention de r88/ saus causer aux liers les dommages intolérables qu'ils avaient pu craindre au début.

Mais précisément parce que l'expérience est faite, on doit se demander si elle ne pourrait pas sans inconvénienl se renouveler chaque hiver de façon à relever un peu l'étiage du Rhône, ou mène être poussée plus loin ct porter sur des écarts de niveau encore plus étendus. C'est une question qui ne saurail être tranchée qu'après une instructicn approfondie, mais quil est permis de poser et dont la solution ne semble pas devoir ètre laissée à la Suisse soule comme en 188' ', mais réglée d'un commun accord par les deux pays, prisque la majeure partie du littoral sud du lac Léman est française. Mais alors même que ce motif décisif ferait défaut, la France ne devrait pas pour cela se désintéresser d'une réglementation qui influe directement sur le régime d'un flenve qui ccule chez elle sur plus de 500 kilomètres.

Il n'cst pas indifférent pour notre pays, en effet, que les basses eaux du Rhône descendant à roo $\mathrm{mc}$./s, ou qu'on les maintienne à $550 \mathrm{mc} / \mathrm{s}$. Dans le premier cas, nos usines perdent une puissance d'étiage très notable, elles en gagnent heaucoup dans le second cas, et la navigation en est également influencée. Je n'ai pas à m'occuper de ce dernier point de vue, mais, sans sortir du domaine de la force hydranlique; je vais montrer l'intérèt que nous pouvons a voir à une meilleure réglementation des niveaux du lac Léman.

I.e régime actnel permet, comme jo l'ai dit, de maintenir le débit d'étiage du Rhône à roo mc. par seconde (roo mc. les dimanches) après la jonction de l'Arve. Comme il ne reçcit aucun affluent notable jusqu'à la frontière française,

(1) La pierre du "Niton " est un gros bloc erratique qui émerge du lac dans le port de Genève. Ainsi nommée, dit-on, d'une ancienne consécration a Neptune, dieu de la mer, ce bloc célèbre porte une plaque métallique qui a servi de base à tous les nivellements de la confédération. C'est le repère du Niton désigné par abréviation P. N. dans les documents teclnnques fédéraux Son altitude. longtemps discutée, serait de $373 \mathrm{~m} .62$ au-dessus du zéro du nivellement général fondamental de la Franre.

(2) On réduit ce débit à 100 mètres cubes le dimanche de paçon à reconstituer partielloment la réserve ce jour-là où les besoins de force motrice sont moins grands que pendant la semaine. 